\title{
Cross-Talk Suppression in High Density Printed Circuit Board Using Magnetic Composite Filled in Spacing between Signal Lines
}

\author{
Shinji Kayama, Makoto Sonehara, Toshiro Sato, Kiyohito Yamasawa, Member, IEEE, \\ Yoshimasa Miura, Fellow, IEEE
}

Spin Device Technology Center, Shinshu University, Nagano 380-8553, Japan

\begin{abstract}
Recently, high density printed circuit board (HD-PCB) with below $50 \mu \mathrm{m} / 50 \mu \mathrm{m}$ line/space has been developed. In the HD-PCB, since the spacing between adjacent signal lines becomes very small, the cross-talk electromagnetic interference among adjacent lines becomes serious. In general, in order to suppress the cross-talk, the ground line is located at the spacing between signal lines because of the decrease of mutual capacitance among adjacent signal lines. The authors have proposed a magnetic method for reducing the cross-talk. The composite magnetic material was filled in the spacing between signal lines instead of the ground line. The magnetic composite composed of Fe-Si-B-Cr amorphous particles with a mean diameter of $6 \mu \mathrm{m}$ and epoxy resin. From the experiments using the PCB-TEG with a $130 \mu \mathrm{m} / 50 \mu \mathrm{m}$ line/space and $25 \mathrm{~mm}$ line length, the cross-talk was suppressed in the wide frequency range by using magnetic composite. In case of using 51 vol. \% amorphous composite, the cross-talk suppression was up to $20 \mathrm{~dB}$ around $1 \mathrm{GHz}$. The influence of the magnetic composite on the transmission and reflection in the main signal line was very small. Therefore, the proposed method is effective for cross-talk suppression without influencing on the signal transmission.
\end{abstract}

Index Terms-HD-PCB, cross-talk, magnetic composite, Fe-based amorphous particle

\section{INTRODUCTION}

$\mathrm{O}^{\mathrm{s}}$ PERATION frequency of the electronic apparatus has been increased, where the high density printed circuit board (HD-PCB) has been used for mother-board. Recently, the HD-PCB with below $50 \mu \mathrm{m} / 50 \mu \mathrm{m}$ line/space has been developed. In the HD-PCB, since the spacing between adjacent signal lines becomes very small, the cross-talk electromagnetic interference (EMI) among adjacent lines becomes serious [1], [2]. Fig. 1 shows the origin of the crosstalk problem. Both mutual-inductance $M_{\mathrm{ij}}$ and mutualcapacitance $C_{\mathrm{ij}}$ between parallel signal lines are shown in the figure. In general, in order to suppress the cross-talk, the ground line is located at spacing between signal lines [3] and the influence of mutual capacitance $C_{\mathrm{ij}}$ becomes small. However, the ground line method cannot suppress the crosstalk owing to the mutual inductance.

The authors have proposed a magnetic method for reducing the cross-talk using the composite magnetic material filled in the spacing between signal lines shown in Fig. 2, instead of the ground line. The authors already clarified the effect of such magnetic method on the cross-talk suppression using FDTD simulation [4]. Since the proposed method does not use the ground line between signal lines, higher density PCB with a very narrow spacing can be realized.

This paper describes the experimental investigation of the cross-talk suppression using Fe-based amorphous composite filled in the spacing between parallel signal lines.

Manuscript received March 05, 2009. Current version published September 18, 2009. Corresponding author: T. Sato (e-mail:sato@yslab.shinshu-u.ac.jp). Digital Object Identifier 10.1109/TMAG.2009.2021406

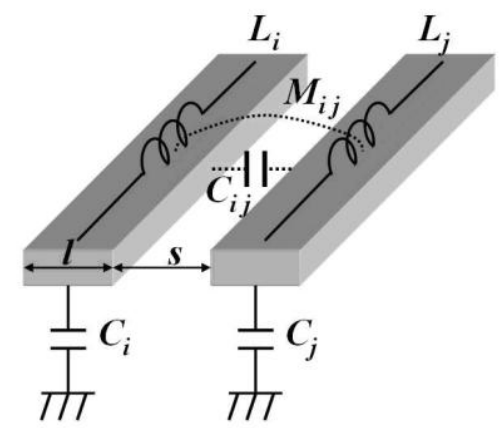

Fig. 1 Origin of cross-talk between adjacent signal lines.

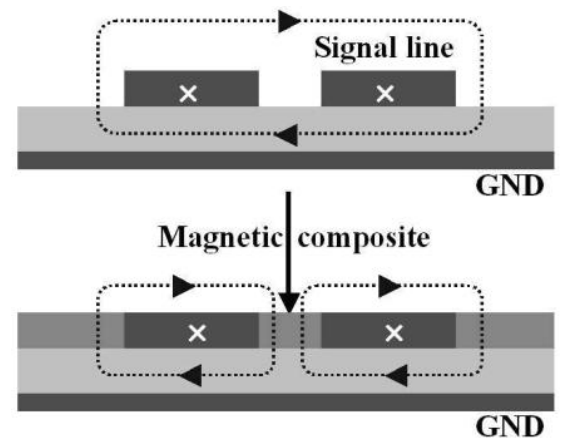

Fig. 2 Magnetic method for cross-talk suppression.

\section{EXPERIMENTAL PROCEDURE}

\section{A. Magnetic composite material and magnetic properties}

The magnetic composite was made by screen printing of the composite paste consisting of the Fe-based amorphous particles and the epoxy resin. In the silica-coated Fe-Si-B-Cr amorphous particle used in the composite, a mean particle diameter was about $6 \mu \mathrm{m}$ and the saturation magnetization was $1.32 \mathrm{~T}$. Fig. 3 shows a photograph and particle size 


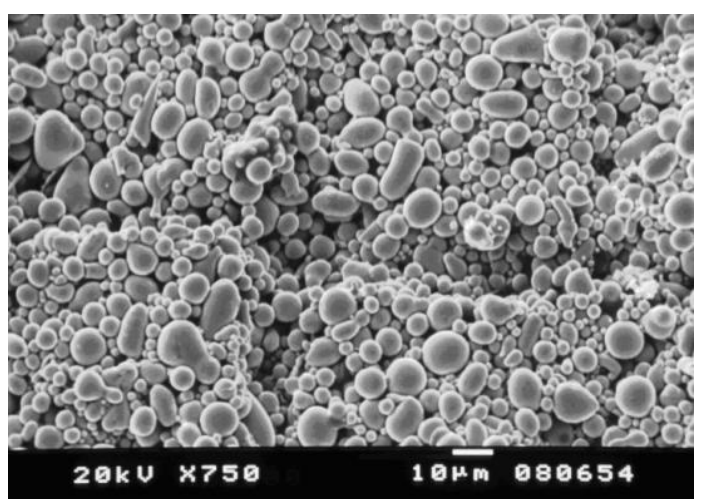

(a) Photograph

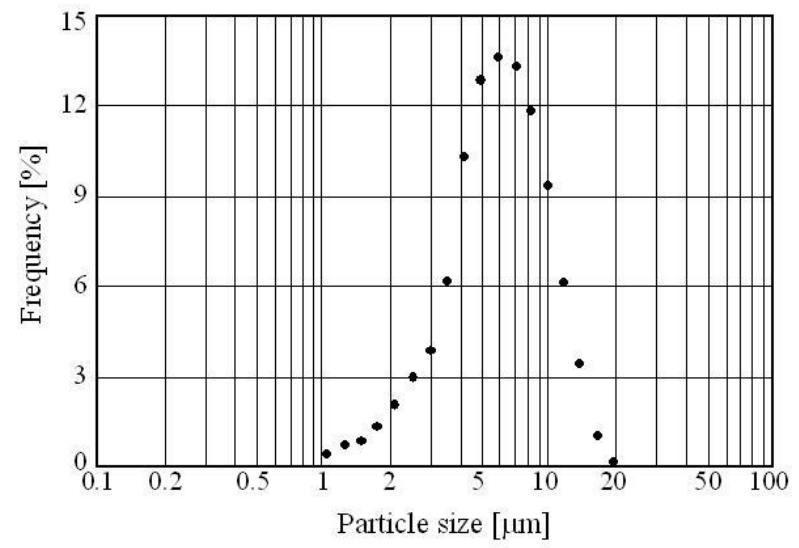

(b) Particle diameter distribution

Fig. 3 Photograph and particle size distribution of Fe-Si-B-Cr amorphous powder made by spinning water atomization process (SWAP) [5].

distribution of the Fe-Si-B-Cr amorphous powder made by spinning water atomization process (SWAP) [5]. The particle shape was close to the sphere.

The post curing temperature after screen printing was about

$450 \mathrm{~K}$. The two types of the composites were fabricated, one was $49 \mathrm{vol} . \%$ amorphous composite, the other was $51 \mathrm{vol} . \%$ amorphous composite.

Fig. 4 shows the magnetic properties of the two types of the magnetic composites. As shown in Fig. 4(a) indicating the static magnetization curve measured by vibrating sample magnetometer, the saturation magnetization $M_{\mathrm{s}}$ of the composites was about 0.65 T. Fig. 4(b) shows the complex permeability vs. frequency measured by coaxial method. In the 49 vol.\% amorphous composite, the low frequency real part of permeability was 8 , and that of 51 vol. $\%$ amorphous composite was slightly higher value of 9 . Though not shown here, the electrical resistivity of the magnetic composite was about $10^{3} \Omega \mathrm{m}$.

B. Filling of magnetic composite into the spacing between signal lines

The PCB with $130 \mu \mathrm{m} / 50 \mu \mathrm{m}$ line/space and $25 \mathrm{~mm}$ line length was used for the experiments, and its designed

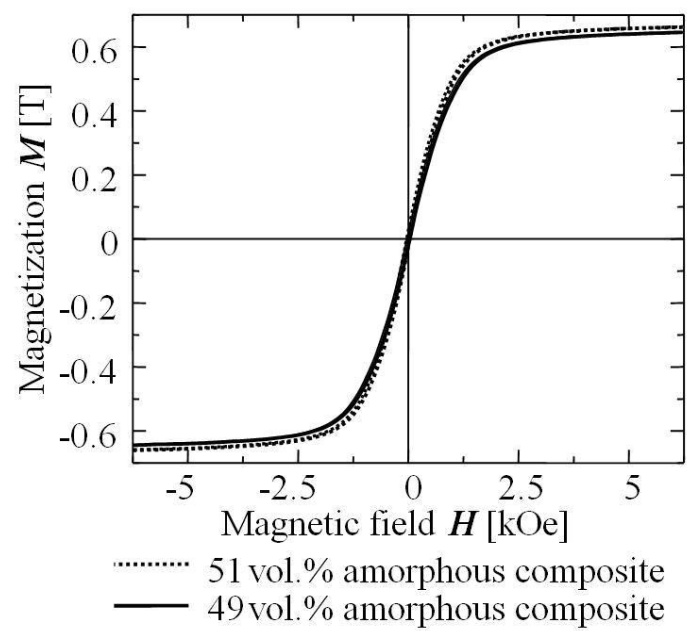

(a) Static magnetization curve

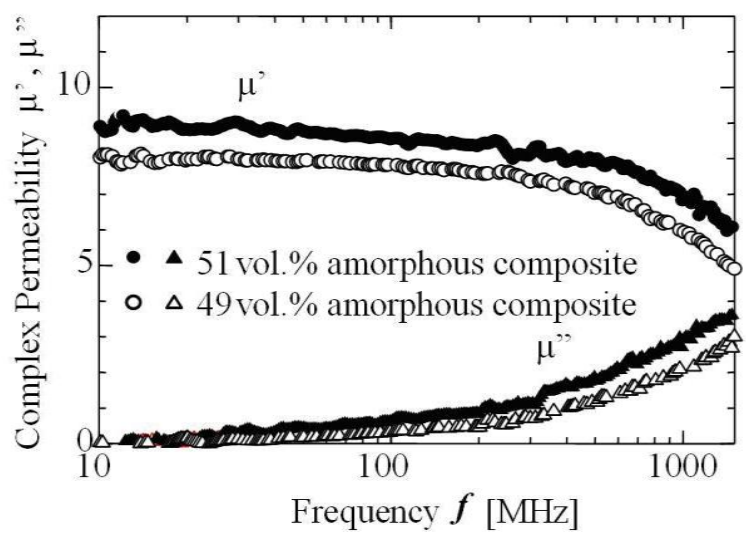

(b) Complex permeability vs. frequency

Fig. 4 Magnetic properties of two types of composite magnetic materials with different amorphous volume ratio.

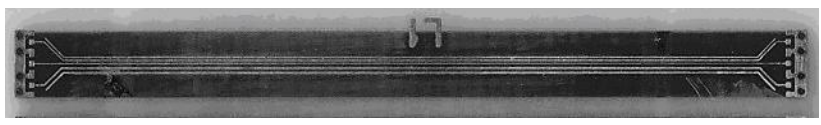

Fig. 5 PCB-TEG sample used in experiments.

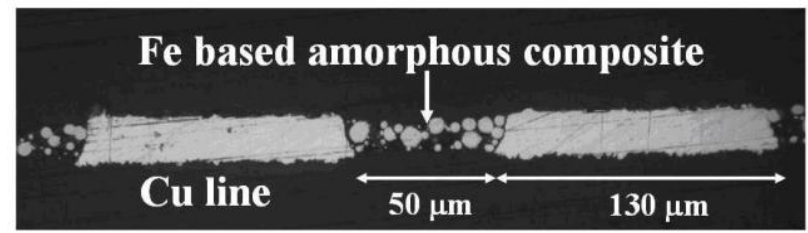

Fig. 6 cross-sectional photograph of the magnetic composite filled in the spacing.

characteristic impedance was $50 \Omega$. An example of the PCBTEG sample used is shown in Fig. 5.

The composite paste was printed directly on the top surface of the PCB without solder resist, and then, the post curing 
was done in air, at $450 \mathrm{~K}$ in temperature, for 1 hour. In order to remove the cured composite on the top surface of the signal

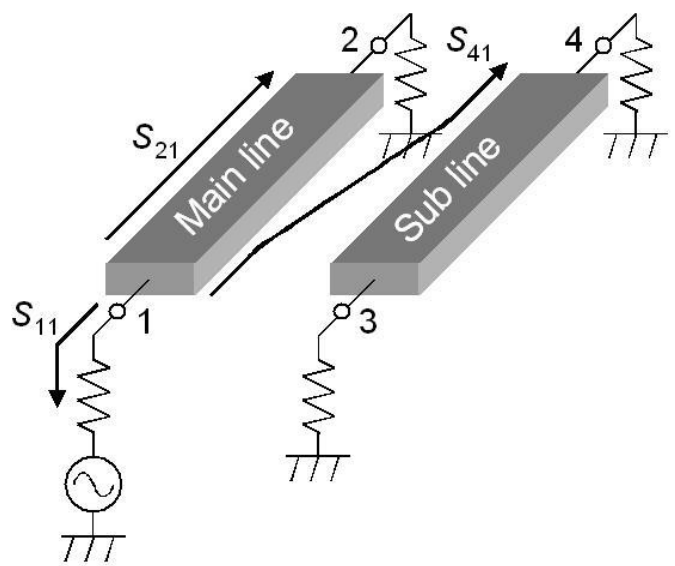

Fig. 7 Definition of the cross-talk in the PCB-TEG sample under test.

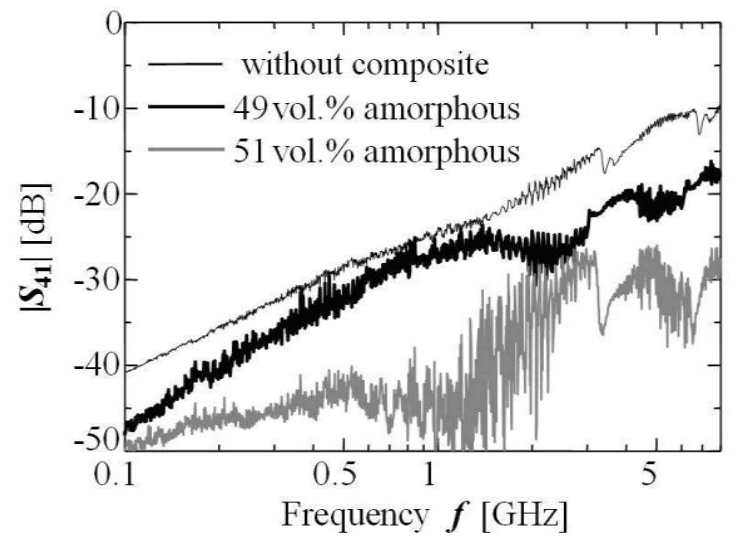

Fig. 8 Cross-talk $S_{41}$ from main line to sub line versus frequency.

lines, the top surface polishing was done after curing process.

Fig. 6 shows the cross-sectional photograph of the magnetic composite filled in the spacing.

\section{Measurement of cross-talk between parallel signal lines}

In order to evaluate the cross-talk between parallel signal lines in the PCB-TEG sample, 4-port network analyzer (Advantest; R3767CG) was used. As shown in Fig. 7, the authors defined the cross-talk from main line to sub line as the scattering parameter $S_{41}$.

Other scattering parameters such as $S_{21}$ and $S_{11}$ were also evaluated, because $S_{21}$ and $S_{11}$ are very important parameters in the main line. Both insertion loss $\left(S_{21}\right)$ and reflection $\left(S_{11}\right)$ in the main line should be as small as possible even when the magnetic composite is used for cross-talk suppression.

\section{ReSUltS AND DISCUSSION}

\section{A. Cross-talk from main line to sub line}

Fig. 8 shows the scattering parameter $S_{41}$ from main line to sub line $v s$. frequency. In the figure, thin solid line shows the result obtained for the reference PCB-TEG without magnetic

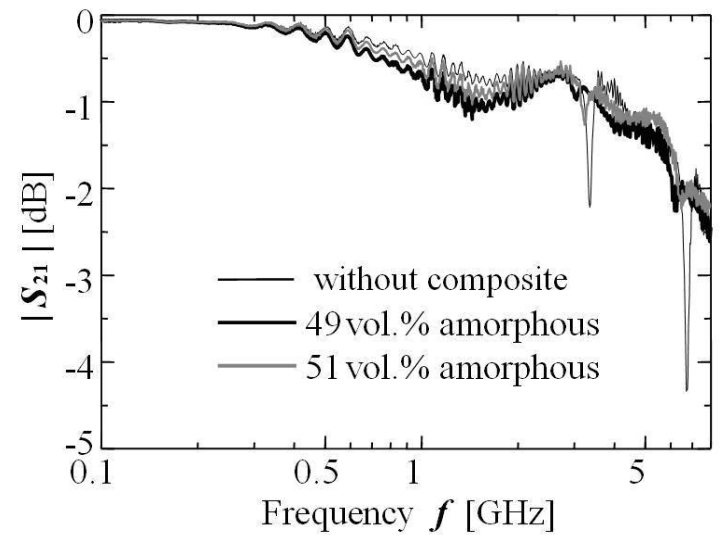

(a) Transmission $S_{21}$

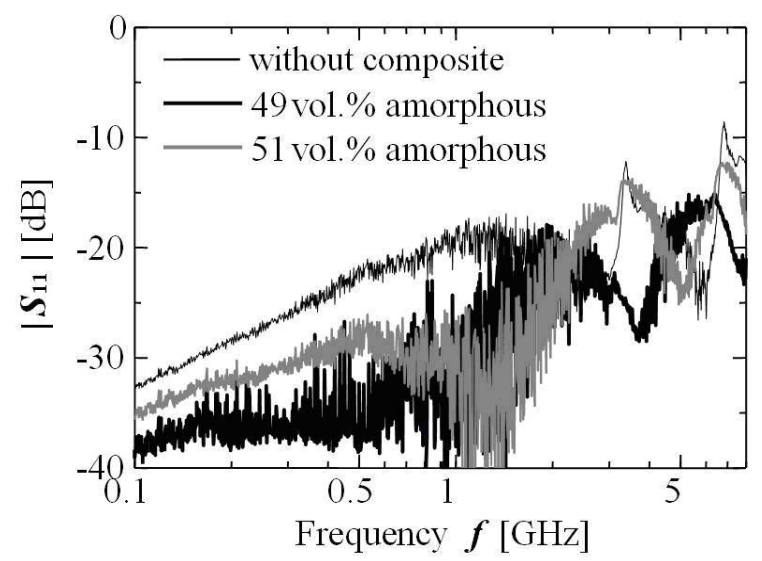

(b) Reflection $S_{11}$

Fig. 9 Transmission $S_{21}$ and reflection $S_{11}$ in the main line versus frequency.

composite. Thick solid line shows the $S_{41}$ obtained for PCBTEG including 49 vol. $\%$ amorphous composite filled in the spacing. The gray line is in case of using 51 vol.\% amorphous composite. From the results of $S_{41}$, the cross-talk was suppressed in the wide frequency range by using magnetic composite. In case of using 49 vol.\% amorphous composite, the cross-talk suppression was $3-10 \mathrm{~dB}$. In case of using 51 vol.\% amorphous composite, on the other hand, the cross-talk suppression was 10-20dB. As shown in Fig. 4, the difference between magnetic properties of 49 vol. $\%$ and 51 vol.\% amorphous composite is relatively small. The large difference in the cross-talk suppression using different volume ratio composite may be due to the top surface polishing process for the cured composite, that is, weak suppression may be owing to the over polishing.

\section{B. Insertion loss and reflection in the main line}

The influence of the magnetic composite on the signal transmission and reflection in the main line was evaluated, because these are very important issue for the main line. 
Fig. 9 shows the transmission coefficient $S_{21}$ and reflection coefficient $S_{11}$ measured for different three kinds of PCBTEGs. From the results, the influence of the magnetic composite on the transmission coefficient $S_{21}$ was very small. Therefore, it is considered that the excess insertion loss due to the magnetic composite is negligible small. The reflection coefficient $S_{11}$ measured for the three kinds of PCB-TEGs was below $-10 \mathrm{~dB}$ in the wide frequency range. The reflection in case of using magnetic composite became smaller than that of the reference PCB-TEG.

Therefore the proposed magnetic method enables to suppress the cross-talk effectively without influencing on the signal transmission.

\section{CONCLUSION}

A magnetic method for reducing the cross-talk in the high density printed circuit board has been proposed and evaluated experimentally using Fe-based amorphous composite filled in the spacing between signal lines. The proposed method is effective for cross-talk suppression without influencing on the signal transmission. Since the proposed method is different from the conventional method using the ground line between signal lines, the new method will be suitable for higher density PCB with a very narrow spacing.

\section{REFERENCES}

[1] Jing Xiaosong, Zhou Runjing, "Crosstalk Analysis and Simulation in High-Speed PCB Design," International Conference on Electronic Measurement and Instruments 2007 (ICEMI '07), pp. 2-437-2-440, Aug. 2007.

[2] Y. Kudo, T. Tobana, T. Sasamori, "A study of crosstalk and its suppression between microstrip-lines on a small printed circuit board," IEICE Trans. Commun., Vol.92, No.1, pp.296-303, Jan. 2009.

[3] T. Maeno, Y. Sakurai, T. Unou, K. Ichikawa, O. Fujiwara, "Slit Effect of Common Ground Patterns in Affecting Cross-Talk Noise between Two Parallel Signal Traces on Printed Circuit Boards," IEEJ Trans. Fund. Mater., Vol.128, No.11, pp.657-662, Nov. 2008.

[4] S. Matsushita, M. Natsume, T. Sato, K. Yamasawa, Y. Miura, "Fundamental Investigation on Crosstalk Suppression in High-Density Wired PCB using Magnetic Material," Annual Conference on Magnetics, Magn. Soc. of Japan., 11pB-2, Sep. 2007.

[5] I. Endo, I. Otsuka, R. Okuno, A. Shintani, M. Yoshino, M. Yagi, "Febased amorphous soft-magnetic powder produced by spinning water atomization process (SWAP)," IEEE Trans. Magn., Vol.35, No.5, pp.3385-3387, Sep. 1999. 